Article

\title{
Liquid Gradient Refractive Index Microlens for Dynamically Adjusting the Beam Focusing
}

\author{
Zichun Le *, Yunli Sun and Ying Du \\ Received: 23 September 2015; Accepted: 7 December 2015; Published: 10 December 2015 \\ Academic Editors: Shih-Kang Fan and Nam-Trung Nguyen \\ College of Sciences, Zhejiang University of Technology, Hangzhou 310023, China; sy1785809@sina.com (Y.S.); \\ duying@zjut.edu.cn (Y.D.) \\ * Correspondance: lzc@zjut.edu.cn; Tel.: +086-571-85290552
}

\begin{abstract}
An in-plane liquid gradient index (L-GRIN) microlens is designed for dynamically adjusting the beam focusing. The ethylene glycol solution (core liquid) withde-ionized (DI) water (cladding liquid) is co-injected into the lens chamber to form a gradient refractive index profile. The influences of the diffusion coefficient, mass fraction of ethylene glycol and flow rate of liquids on the refractive index profile of L-GRIN microlens are analyzed, and the finite element method and ray tracing method are used to simulate the convection-diffusion process and beam focusing process, which is helpful for the prediction of focusing effects and manipulation of the device. It is found that not only the focal length but the focal spot of the output beam can be adjusted by the diffusion coefficient, mass fraction and flow rate of liquids. The focal length of the microlens varies from 942 to $11 \mu \mathrm{m}$ when the mass fraction of the ethylene glycol solution varies from 0.05 to 0.4 , and the focal length changes from 127.1 to $8 \mu \mathrm{m}$ by varying the flow rate of the core liquid from $0.5 \times 10^{3}$ to $5 \times 10^{3} \mathrm{pL} / \mathrm{s}$ when there is no slip between the core and cladding inlet. The multiple adjustable microlens with a simple planar microfluidic structure can be used in integrated optics and lab-on-chip systems.
\end{abstract}

Keywords: in-plane liquid gradient index (L-GRIN) microlens; optofluidics waveguide; adjustable focal length; convection-diffusion process; beam focusing; finite element method

\section{Introduction}

Tunable microlenses are widely used in microfluidic or lab-on-chip systems [1] due to their fine tuning of the light. A tunable microlens adaptively reshapes the input light including adjusting its focal position, intensity, beam profile, and even propagation direction [2]. They can also be used for ordinary coupling, collimating and focusing, and in wide range of lab-on-a-chip applications such as flow cytometry [3-5], on-chip optical tweezers and single molecule detection [6,7].

Several kinds of tunable microlenses are developed for providing adaptive focusing with adjustable curved refractive surfaces [2], such as a micromeniscus surface actuated by electro-wetting [8], liquid microlenses based on the dielectrophoretic effect [9-11], microlenses based on the thermal effect [12], or hydraulically actuated polydimethylsiloxane (PDMS) membranes $[13,14]$. Among them, electro-wetting and dielectrophoresis lenses are electrically driven, while other microlenses whose focal lengths are variable are either based on pressure-induced systems using an elastic membrane, or on thermal effect. A mechanical lens whose focal length is controlled by pumping liquid in and out of the lens chamber caused great interest. The operation mechanism of this lens is simple because it only requires a fluid pumping system. There are two working mechanisms which are often used to implement these tunable liquid microlenses. One is to achieve the refraction of light at curved, optically smooth, liquid-liquid interfaces between 
two flowing fluids by controlling laminar flows. Recently, the Whitesides group has developed a dynamically reconfigurable liquid-liquid lens [15] with the convex shape of liquid-liquid interfaces in a microfluidic expansion chamber. The curvatures in the liquid-liquid interfaces were controlled by adjusting hydrodynamic flow conditions, and then adjustable focal length was produced. However, in the liquid-liquid lens, a higher speed of the laminar flows is necessary, which means large amounts of fluid have to be supplied discontinuously for keeping the microlens working. Utilization of so-called liquid gradient refractive index (L-GRIN) microlenses [16] is the other approach and also the preferable way to achieve a tunable microlens. Similarly, with the traditional solid self-focusing lens, the refractive index of the L-GRIN microlens increases continuously from the cladding region to the core optical axis. However, a gradient refractive index of the L-GRIN microlens is achieved by dynamically adjusting hydrodynamic flow conditions rather than by changing the geometrical structure of the lens for the traditional one. In contrast to the liquid-liquid interface-based microlens, the L-GRIN lens operates through the diffusion in multiple flows rather than by relying on a clearly defined, curved liquid-liquid interface. Therefore, the flow consumption rate is much lower in the L-GRIN lens [17]. These lenses focus a light beam through a liquid medium with a two-dimensional (2D) refractive index gradient. The two-dimensional (2D) refractive index profile and, subsequently, the focal length of the L-GRIN lens can be tuned by changing the ratio of the flow rates of the two inlets [17]. However, most L-GRIN microlenses are only capable of out-of-plane focusing. In-plane-focusing tunable microlens are, however, proven to be preferable in integrative devices, which can be fabricated and seamlessly integrated with other on-chip fluidic and optical components such as a lab-on-a-chip lasers [18] and optical waveguides [19]. In particular, it has inspired the creation of a variety of innovative devices controlled by flow rates and liquid compositions, and variable light focusing was measured and shown, but the quantitative relation between liquid parameters and focal length has not been demonstrated clearly. It will lead to the unpredictability of the focusing effect in the device.

In this paper, an in-plane tunable L-GRIN microlens was designed for dynamically adjusting the beam focusing, which can be more readily integrated for lab-on-a-chip applications. The convection-diffusion process of liquids in the microfluidic chamber is firstly simulated with the finite element method (FEM) when a high-refractive-index solution is injected side-by-side into a low-refractive-index solution. During the convection and diffusing process, the diffusion profile of the liquids, and hence the refractive index profile within the L-GRIN microlens, varies with the hydrodynamic flow conditions. Thus, the refractive index profile of the L-GRIN microlens is calculated and discussed numerically under different flow conditions, and the beam transmission and focusing process in the L-GRIN microlens is simulated using the ray tracing method. The effects of the diffusion coefficient, mass fraction and flow rate of liquids on the input beam focusing effect, including the focal length and the size of the focal spot, were demonstrated. Therefore, an in-plane tunable beam focusing is achieved using a simple planar microfluidic structure; in addition, with the simulation of the convection and diffusing process, the ray tracing method gives us approaches to predict the focusing effect of an L-GRIN microlens.

\section{Structure and Principle of L-GRIN Micrlens}

The schematic of the L-GRIN microlens designed is shown in Figure 1. The structure of the microlens as shown in Figure 1a consists of the microfluid chamber, core inlet, cladding inlet and outlet. The core liquid and the cladding liquid are injected through the core inlet and cladding inlet, respectively, which flow out through the outlet. $H_{\text {in_core }}, W_{\text {in_core, }}, H_{\text {in_clad }}, W_{\text {in_clad }}, H_{\text {out }}, W_{\text {out }}$ represent the height and width of the core inlet, cladding inlet and outlet, respectively. The main part of the L-GRIN microlens is a micro-cylindrical chamber, where the diffusion and convection process of liquids occurs and the gradient refractive index profile appears. The xoy cross-sectional view of the L-GRIN microlens together with the substrate is also illustrated in Figure $1 \mathrm{a}$, where $d_{\text {core }}$ which is designed to be $50 \mu \mathrm{m}$ stands for the diameter of the core inlet. Similarly, $d_{\text {clad }}$ is the diameter of the 
cladding inlet and is designed to be $150 \mu \mathrm{m}$. In the chamber, ethylene glycolsolution (core liquid) is injected side-by-side into de-ionized (DI) water (cladding liquid) from the same direction as shown in Figure 1b. On account of the well-known manner in which the refractive index of liquids varies with the wavelength of incident light, in our simulation, the wavelength of incident light is set to be 500nm without considering the dispersion of light. The diffusion and convection process between ethylene glycol solution and DI water results in a 2D refractive index profile in the lens chamber, as shown in the left graph of Figure 1c.An axis-symmetric refractive index gradient is accordingly achieved in the xoy plane and the simulation result is given in the right graph of Figure 1c, which shows that the refractive index of the L-GRIN microlens increases continuously from the cladding region to the core optical axis. The maximum refractive index comes near the inlet, and under the influence of diffusion, the refractive index decreases along the flowing direction of the liquids.

(a)

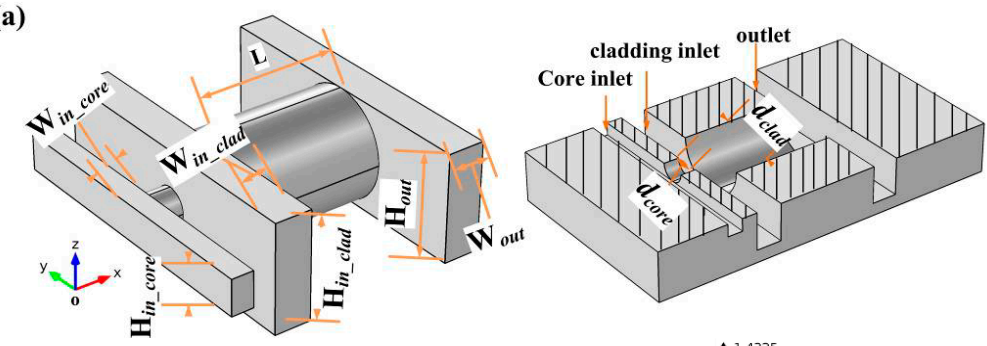

(b)
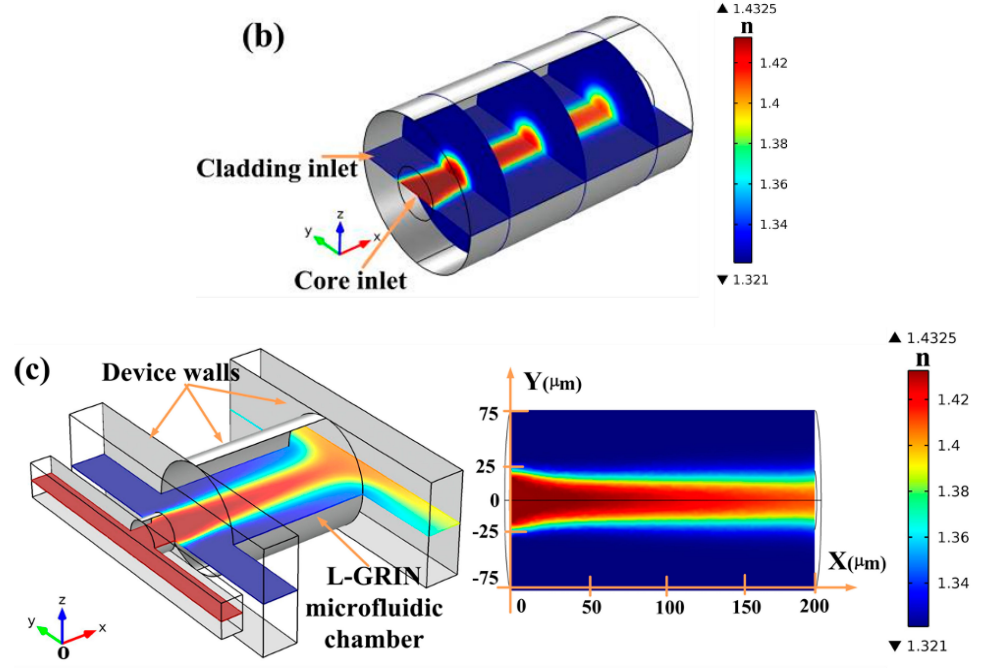

Figure 1. (a) Structure diagram of the tunable L-GRIN microlens (left graph), in which $H_{\text {in_core }}=50 \mu \mathrm{m}, W_{\text {in_core }}=30 \mu \mathrm{m}, H_{\text {in_clad }}=150 \mu \mathrm{m}, W_{\text {in_clad }}=50 \mu \mathrm{m}, H_{\text {out }}=150 \mu \mathrm{m}, W_{\text {out }}=50 \mu \mathrm{m}$. The xoy cross-sectional view of the L-GRIN microlens together with the substrate (right graph), in which $d_{\text {core }}=50 \mu \mathrm{m}, d_{\text {clad }}=150 \mu \mathrm{m}$; (b)The simplified model of the microfluidic chamber; (c) Simulated refractive index profile in the microfluidic chamber (left graph) and the refractive index profile in the xoy plane (right graph).

To simulate and optimize the refractive index profile and light propagation in the L-GRIN microlens under different conditions, the FEM and optical ray tracing method are adopted. The refractive index profile can be calculated by simulating the diffusion and convection process of liquids in the microfluidic chamber. When the refractive index of liquids is mainly affected by diffusion, the process can be described by Fick's second law:

$$
\frac{\partial C}{\partial t}=D \nabla^{2} C
$$


In which $C$ is the solution concentration and $t$ is the diffusion time. $D$ represents the diffusion coefficient that may determine the diffusion speed between the core liquid and the cladding liquid. As shown in Equation (1), the solution concentration changes with time and space. However, in the microfluidic chamber, the diffusion and the convection have a combined effect on the refractive index profile of the L-GRIN microlens. The convective-diffusive process in the chamber is therefore rewritten as [20]:

$$
\frac{\partial C}{\partial t}=D \nabla^{2} C-U \nabla C
$$

where $U$ is defined to be the average flow velocity in the chamber. For a steady state flow, the distribution of concentration in the device does not vary with time, which means that $\partial C / \partial t=0$. Considering the symmetry of the lens structure, the convective-diffusive process can be simplified as:

$$
D\left(\frac{\partial^{2} C}{\partial x^{2}}+\frac{\partial^{2} C}{\partial y^{2}}\right)-U\left(\frac{\partial C}{\partial x}+\frac{\partial C}{\partial y}\right)=0
$$

The concentration distribution for the full, developed, steady-state flow can be expressed as Equation (4) [21].

$$
c^{\prime}\left(x^{\prime}, y^{\prime}\right)=r+\frac{2}{\pi} \sum_{n=1}^{\infty} \frac{\sin (\pi n r)}{n} \cos \left(n \pi y^{\prime}\right) \exp \left[\frac{1}{2}\left(P e-\sqrt{P e^{2}+4 n^{2} \pi^{2}}\right) x^{\prime}\right]
$$

In the normalized coordinate system, $x^{\prime}=x / R, y^{\prime}=y / R$, where $R$ is the radius of the microfluidic chamber. Then $c^{\prime}=c / C_{0}$ is the normalized concentration, and $C_{0}$ stands for the initial concentration. $P e$ is called the Peclet number, which is a dimensionless number and represents the relative proportion of convection to diffusion. $P e$ is proportional to the laminar velocity across the transversal section of the microfluidic chamber and the length of the microfluidic chamber, and $P e$ is also inversely proportional to the diffusion coefficient. $P e$ is usually used to describe the coefficient involved in the convection and diffusion process for a common analysis. However, the effect caused by convection and diffusion is analyzed separately in the present paper in order to dynamically reshape the light beam of the L-GRIN microlens.

For the structure we designed, $U=\left(Q_{\text {core }}+Q_{\text {clad }}\right) / R^{2} \pi$ is defined as the average velocity, where $Q_{\text {core }}$ is the flow rate of the core liquid and $Q_{\text {core }}$ is the flow rate of the cladding liquid; $r$ is the normalized initial boundary ratio between the core and cladding liquids and is expressed as [22]:

$$
\alpha \beta^{2}(r-1)^{4}+r(r-1)\left[r^{2}+3 r-\alpha(r-1)(r-4)\right] \beta-r^{4}=0
$$

where $\alpha=Q_{\text {core }} / Q_{\text {clad }}, \beta=\mu_{\text {core }} / \mu_{\text {clad }}$, and $\mu_{\text {core }}$ is the liquid viscosity of the core liquid and $\mu_{\text {clad }}$ is the liquid viscosity of the cladding liquid. The initial parameters are set as $\left.c^{\prime}\right|_{x^{\prime}=0,0 \leqslant y^{\prime}<r=1}=1$, $\left.c^{\prime}\right|_{x^{\prime}=0, y^{\prime}=r}=r$ and $\left.c^{\prime}\right|_{x^{\prime}=0, r \leqslant y^{\prime} \leqslant 1}=0$, in which $\left.\frac{\partial c^{\prime}}{\partial y^{\prime}}\right|_{x=\infty, 0 \leqslant y^{\prime} \leqslant 1}=0$ stands for the full mixing state at the outlet and $\left.\frac{\partial c^{\prime}}{\partial y^{\prime}}\right|_{y=1}=0$ represents the full mixing state at the chamber walls.

Once the liquids for the core and the cladding liquids are determined, the liquid viscosity and diffusion coefficient are considered to be constant. The position of the focal point can be tuned along the $x$-direction by changing the refractive index profile, which can be achieved by adjusting the flow rate of the core or cladding liquid. According to Equation (2), if the average velocity $U$ is large, the diffusion in the xoy plane can be neglected and only the refractive index profile in the yoz plane needs to be calculated, where the plane is perpendicular to the liquid flow direction. On the contrary, when the average velocity $U$ is small, the diffusion in both $x$ and $y$ directions has to be discussed. The refractive index profile can be obtained according to the concentration profile [23].

$$
n\left(x^{\prime}, y^{\prime}\right)=c_{\text {core }}^{\prime}\left(x^{\prime}, y^{\prime}\right) n_{\text {core }}+c_{\text {clad }}^{\prime}\left(x^{\prime}, y^{\prime}\right) n_{\text {clad }}
$$


where $n\left(x^{\prime}, y^{\prime}\right)$ is the refractive index profile of the L-GRIN microlens in the $x^{\prime}$ oy' plane, and $c_{\text {core }}^{\prime}$ and $c_{\text {clad }}^{\prime}$ are the normalized concentration profile of the core and cladding liquids in the $x^{\prime} o y^{\prime}$ plane, respectively. The focusing of the input light beam through the microlens is determined by the refractive index profile.

Because of the decisive effect of the convection-diffusion process on the refractive index profile of the L-GRIN microlens, the average velocity $U$ and the diffusion coefficient $D$, which are also found to vary with the concentration $C$ and temperature, are important parameters for tunable focusing. For example, the diffusion coefficients between DI water and ethylene glycol are $3.75 \times 10^{-10}$ and $1.17 \times 10^{-9} \mathrm{~m}^{2} / \mathrm{s}$ with different mass fractions of 0.025 and 0.95 for ethylene glycol [24]. In addition, when the temperature varies from 30 to $50^{\circ} \mathrm{C}$, the diffusion coefficient changes from $3.15 \times 10^{-10}$ to $6.45 \times 10^{-10} \mathrm{~m}^{2} / \mathrm{s}$ with a fixed mass fraction of 0.8 for the ethylene glycol [25]. Due to the temperature of liquids usually being stationary during the convection-diffusion process, thediffusion coefficient $D$, concentration $C$ and average velocity $U$ of liquids are the main influence factors for adjusting the focusing performance of the L-GRIN microlens. It was found by simulations as follows that an adjustable focal length could be achieved under a relatively lower average velocity while the size of the focal spot had to be tuned at a high enough average velocity. Therefore, both the focal length and the focal spot of the output beam can be adjusted by carefully controlling the average velocity of the liquids. Suppose that the DI water and ethylene glycol solution are chosen as the cladding and the core liquids, respectively, and two cases of the average velocity with relative slip and without relative slip between the core and cladding inlet are disscused. The subsequent discussion will be focused on how the adjustment of the output beam will be implemented by adjusting these influence factors.

In order to validate our numerical simulation, the comparison between the observed light propagation in Reference [23] and our simulation results was illustrated in Figure 2. Figure 2a shows light propagation in the optofluidic waveguides under different fluidic conditions, and Figure $2 b$ shows our simulation results at the same fluidic conditions. When driven at a highflow rate of $Q_{\text {clad }}=50 \mu \mathrm{L} \cdot \min ^{-1}(P e=0.07)$, the light propagates in a straight line and shows no focusing effect. In contrast, at a low flow rate of $Q_{\text {clad }}=0.5 \mu \mathrm{L} \cdot \mathrm{min}^{-1}$, the light exhibits curved-ray trajectories and converges repeatedly for different core widths, $r=1 / 6,1 / 3$ and $1 / 2(P e=0.0005,0.0007$ and 0.001 , respectively) [23].
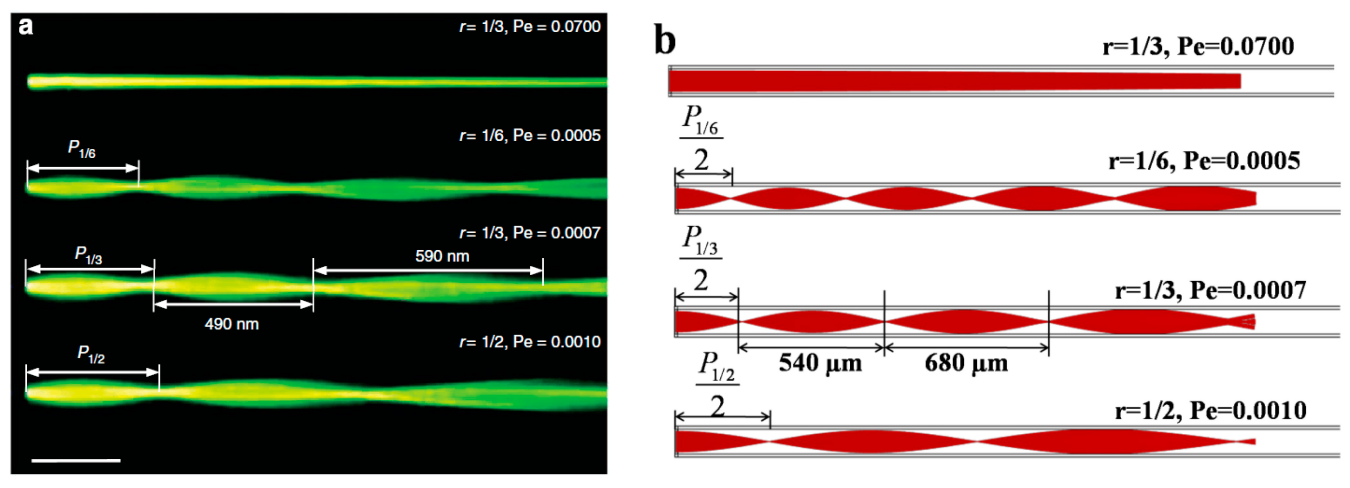

Figure 2. (a) Observed light propagation under different flow conditions [23]. At a high flow rate of $Q_{\text {clad }}=50 \mu \mathrm{L} \cdot \mathrm{min}^{-1}$, the light is confined in the core due to the step-index distribution. At $Q_{\text {clad }}=0.5 \mu \mathrm{L} \cdot \mathrm{min}^{-1}$, the diffusion-induced gradient of the refractive index causes the light to repeatedly merge. With increasing $r$, the length of the first section becomes larger, for example $P_{1 / 6}=270 \mu \mathrm{m}, P_{1 / 3}=300 \mu \mathrm{m}$ and $P_{1 / 2}=340 \mu \mathrm{m}$. The focusing period also increases; for example, the first period for $P_{1 / 3}$ is $300 \mu \mathrm{m}$, the second is extended to $490 \mu \mathrm{m}$ and the third is $590 \mu \mathrm{m}$. (Scale bar equals $300 \mu \mathrm{m}$.)(b) Simulated light propagation under the same flow conditions. With increasing $r$, the length of the first section becomes larger, for example, $P_{1 / 6}=300 \mu \mathrm{m}, P_{1 / 3}=360 \mu \mathrm{m}$ and $P_{1 / 2}=410 \mu \mathrm{m}$. The focusing period also increases; for example, the first period for $P_{1 / 3}$ is $360 \mu \mathrm{m}$, the second is extended to $540 \mu \mathrm{m}$ and the third is $680 \mu \mathrm{m}$. 
Similarly, the length of the first section increases with the core width and decreases almost linearly with the flow rate of the core fluid in both figures. However, when examining the light propagation pattern in detail, it is found that the self-focusing period is chirped such that the focusing period increases. For instance, as shown in Figure 2a, the first period for $P_{1 / 3}$ is $300 \mu \mathrm{m}$, the second is extended to $490 \mu \mathrm{m}$, and the third is $590 \mu \mathrm{m}$, owing to the diminishing bidirectional gradient contrast downstream as a result of diffusion [23]. Furthermore, the first period for $P_{1 / 3}$ is $360 \mu \mathrm{m}$, the second is extended to $540 \mu \mathrm{m}$, and the third is $680 \mu \mathrm{m}$ in Figure $2 \mathrm{~b}$. The small difference in focus length maybe due to the difference between two structures, and the change trends are the same. The match between the experimental results in Reference [23] and our simulation results illustrated that the follow-up simulations could be trusted.

\section{Results and Discussion}

\subsection{TheRefractive Index Profile of the L-GRIN Microlens}

In order to form the gradient refractive index profile in the lens chamber, the ethylene glycolsolution $\left(n_{\text {core }}=1.432\right)$ and DI water $\left(n_{\text {clad }}=1.332\right)$ are co-injected into the lens chamber from the same direction. Once contacting DI water, the ethylene glycol starts to diffuse from the ethylene glycol solution into the DI water. The average velocity $U$ is calculated according to $U=\left(Q_{\text {core }}+Q_{\text {clad }}\right) / R^{2} \pi$. For the purpose of maintaining the same flow rate of the ethylene glycol solution and the DI water (supposingthere is norelative slip between the core and cladding liquids), $Q_{\text {clad }} / Q_{\text {core }}=8$ should be satisfied because the cross-sectional area of the cladding inlet is eight times as large as that of the core inlet. In order to illustrate the influence of the diffusion coefficient $D$ on the refractive index profile of the L-GRIN microlens, the refractive index profiles in the xoy plane were simulated at two different diffusion coefficients $\left(D=1 \times 10^{-9} \mathrm{~m}^{2} / \mathrm{s}\right.$ and $\left.D=4 \times 10^{-10} \mathrm{~m}^{2} / \mathrm{s}\right)$ as shown in Figure 3 . Figure $3 \mathrm{a}, \mathrm{b}$ indicate that the variation of the diffusion coefficient resulting from the variation of the environmental conditions such as temperature has a strong influence on the refractive index profile, and the diffusion phenomenon becomes noticeable with the increase of the diffusion coefficient. This illustrates the fact that the diffusion is a highly activated process for the refractive index profile, provided the flow rates of the liquids are the same.
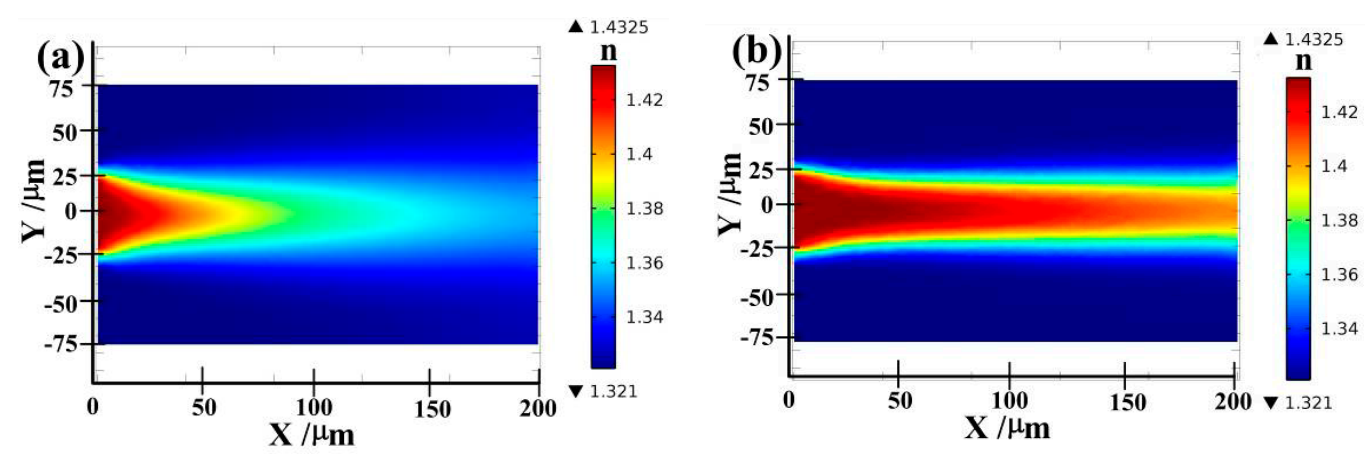

Figure 3. The refractive index profiles in the xoy plane with different diffusion coefficient $D$ (with a fixed mass fraction of 0.3 , and $\left.Q_{\text {clad }}=8, Q_{\text {core }}=8 \times 10^{3} \mathrm{pL} / \mathrm{s}\right) . \quad\left(\right.$ a) $D=1 \times 10^{-9} \mathrm{~m}^{2} / \mathrm{s}$; (b) $D=4 \times 10^{-10} \mathrm{~m}^{2} / \mathrm{s}$.

\subsection{The Adjustment of the Focal Lengthof the L-GRIN Microlens}

It is known that a relatively lower average velocity of liquids offers remarkable diffusion effect, and the focus with an adjustable focal length of the L-GRIN microlens forms by changing the diffusion process. The diffusion process of the liquid is influenced by the average velocity $U$ of liquids and the diffusion coefficient $D$ which is affected simultaneously by the concentration $C$ of the solution. Therefore, the focal length of the L-GRIN microlens can be tuned by adjusting the concentration and 
the flow rateof the liquids. Hereinafter, the concentration of the ethylene glycol solution is altered through changing the mass fraction of the ethylene glycol solution.

\subsubsection{Tuning Mass Fraction of Ethylene Glycol Solution}

Because the diffusion coefficient changes with the concentration of the core liquid, the refractive index profile of the L-GRIN microlens varies with the mass fraction of the ethylene glycol solution. As the diffusion progresses, the solution flowing into different regions has a different concentration, and the diffusion coefficient is, therefore, also different. However, according to Equation (4), compared with the concentrated ethylene glycol solution, the diluted ethylene glycol solution will have an obvious gradient refractive index profile whose diffusion coefficient is larger and can be considered approximately constant during the diffusion process. To facilitate the analysis, smaller mass fractions of ethylene glycol ranging from 0.05 to 0.4 were selected and the focal length of the L-GRIN microlens with different mass fractions was calculated while the flow rate of the liquids was kept the same $\left(Q_{\text {core }}=1 \times 10^{3} \mathrm{pL} / \mathrm{s}, Q_{\text {clad }}=8 \times 10^{3} \mathrm{pL} / \mathrm{s}\right)$. Firstly, we simulated the refractive index profiles of the L-GRIN microlens with the mass fraction increasing from 0.05 to 0.4 by 0.05 , and simulation results showed that the sharper peak profile of the refractive index could be achieved with the increase of the mass fraction. Figure 4a shows the refractive index profile with the mass fraction of 0.2 , and the cross-sectional refractive index profiles at five different locations $(x=50,100$, 150, 200 and $250 \mu \mathrm{m}$ ) are shown in Figure $4 \mathrm{~b}$. The beam transmission and focusing process can be simulated by using the ray tracing method based on the refractive index profile, which is shown in Figure 4c. The focal length is the distance from the outlet to the focal point, and Figure $4 \mathrm{~d}$ shows that the focal length of the L-GRIN microlens can be tuned with different mass fractions of the ethylene glycol solution. When the flow rates of the core and cladding liquid are fixed, the focal length of the L-GRIN microlens varies from 942 to $11 \mu \mathrm{m}$ with a mass fraction of ethylene glycol ranging from 0.05 to 0.4 . Therefore, controlling the mass fraction of the core liquid is a useful way to adjust the focal length of the device.
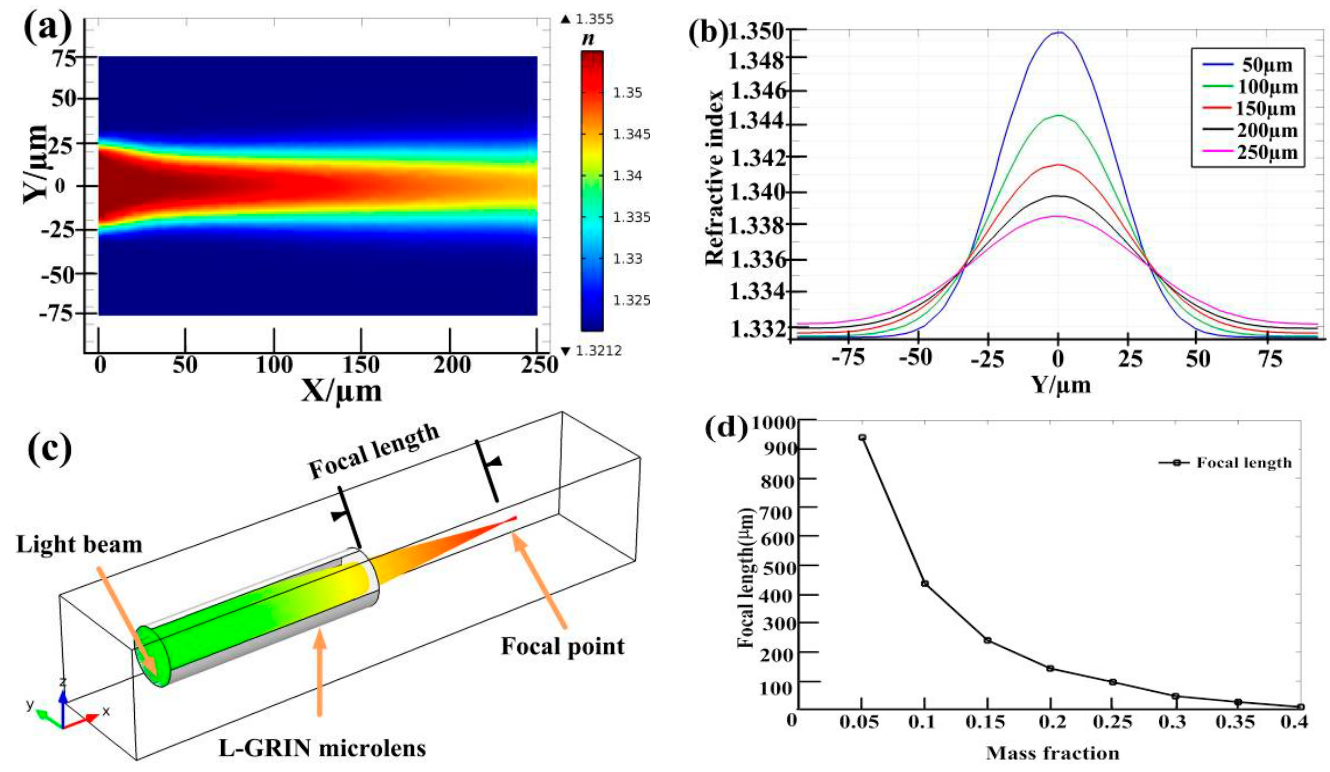

Figure 4. (a) The refractive index profile in the xoy plane with the mass fraction of 0.2 ; (b) The cross-sectional refractive index profiles at five different locations ( $x=50,100,150,200$ and $250 \mu \mathrm{m})$ with $Q_{\text {core }}=1 \times 10^{3} \mathrm{pL} / \mathrm{s}, Q_{\text {clad }}=8 \times 10^{3} \mathrm{pL} / \mathrm{s}$; (c) Beam transmission and focusing process; $(\mathbf{d})$ The focal length of the L-GRIN microlens with different mass fractions of the ethylene glycol solution. 


\subsubsection{Tuning the Flow Rate of Liquids}

The refractive index profile of the L-GRIN microlens can also be tuned by changing the flow rate of the liquids. In order to analyze the influence of the flow rate of the liquids on the focal length of the L-GRIN microlens, the ethylene glycol solution is co-injected with DI water into the lens chamber at the same flow rate ranging from $0.5 \times 10^{3}$ to $5 \times 10^{3} \mathrm{pL} / \mathrm{s}$. In the analysis, we suppose there is no relative slip between the ethylene glycol solution and the DI water. Additionally, for simplifying the calculation, simulations were started by setting $D=8 \times 10^{-10} \mathrm{~m}^{2} / \mathrm{s}$ and $\mu=1 \times 10^{-3} \mathrm{~Pa} \cdot \mathrm{s}$ with a fixed mass fraction of 0.3 .

We simulated the refractive index profiles with the flow rate increasing from $0.5 \times 10^{3}$ to $5 \times 10^{3} \mathrm{pL} / \mathrm{s}$ by $0.5 \times 10^{3} \mathrm{pL} / \mathrm{s}$, and the results demonstrated that the diffusion was no longer dominant with the increase of the flow rate. Figure 5 a shows the refractive index profile with the flow rate of $4 \times 10^{3} \mathrm{pL} / \mathrm{s}$, and the cross-sectional refractive index profiles at five different locations $(x=50$, 100, 150, 200 and $250 \mu \mathrm{m}$ ) are shown in Figure 5b. Figure $5 \mathrm{c}$ shows the focal length of the L-GRIN microlens is adjusted with the different flow rate. According to our calculations, the focal length decreases observably from 128 to $8 \mu \mathrm{m}$ when the flow rate varies from $0.5 \times 10^{3}$ to $5 \times 10^{3} \mathrm{pL} / \mathrm{s}$. However, when the flow rate is larger than $5 \times 10^{3} \mathrm{pL} / \mathrm{s}$, the converging of the light beam caused by focusing becomes more and more unobvious. Therefore, continuously tuning the flow rate of the liquids in a certain range provides a tunable focal length when the mass fraction of the ethylene glycol is kept constant.
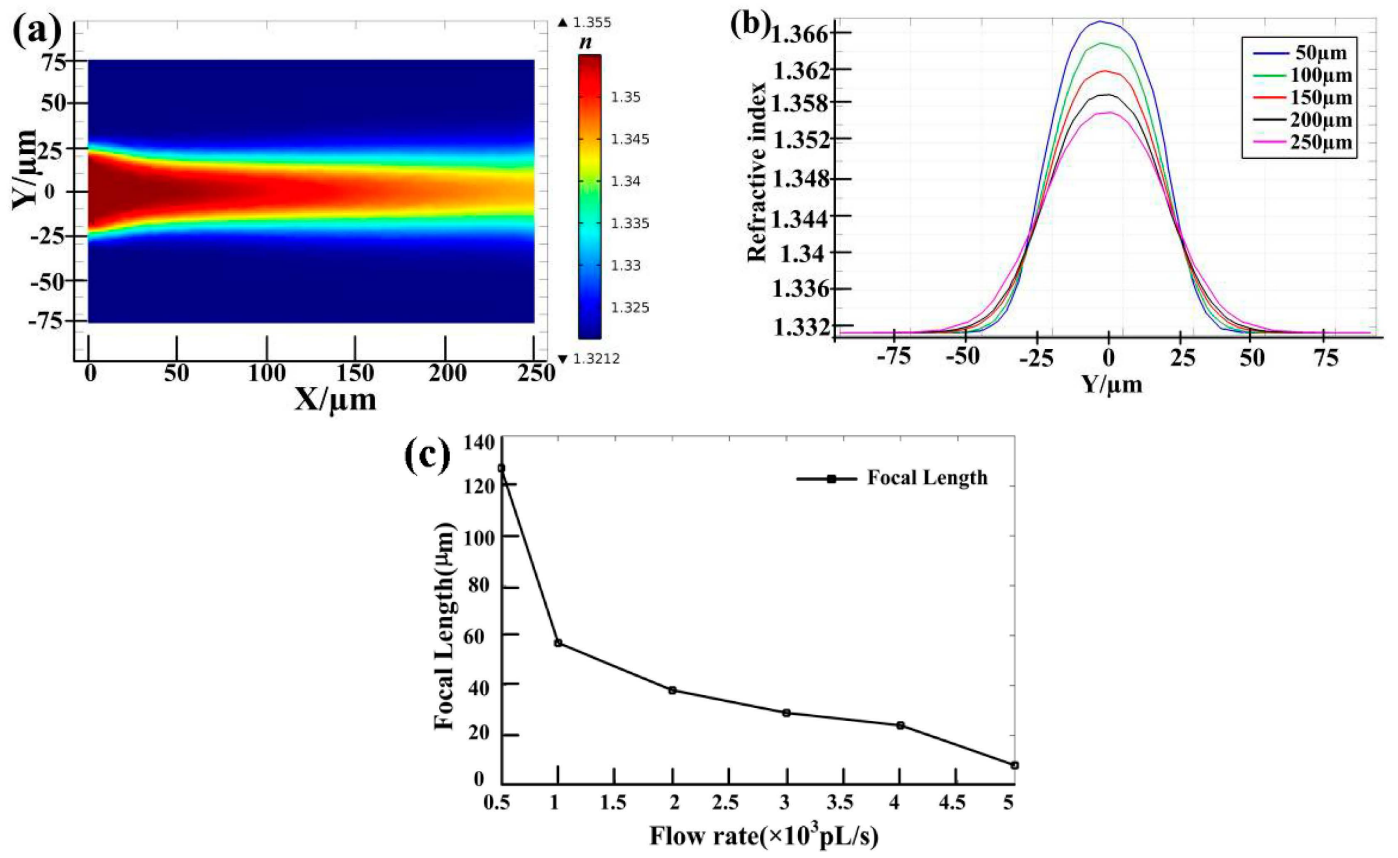

Figure 5. (a) The refractive index profile in the xoy plane with the flow rate of $4 \times 10^{3} \mathrm{pL} / \mathrm{s}$; (b) The cross-sectional refractive index profiles at five different locations $(x=50,100,150,200$ and $250 \mu \mathrm{m})$ with $Q_{\text {clad }}=8 \times 10^{3} \mathrm{pL} / \mathrm{s}, Q_{\text {core }}=1 \times 10^{3} \mathrm{pL} / \mathrm{s}$; (c) The focal length of the L-GRIN microlens with different flow rates of solutions.

In general, the focal length of the lens is related to the lens radius, the refractive index difference between the lens center and border, and the lens thickness; in addition, it is dependent on the wavelength of the incident light which will change the refractive index profile of the lens. Thus, a shorter wavelength of incident light (when other parameters are constant) results in larger refractive index contrast, which causes light to bend toward the lens axis more significantly and leads to the decreased focal length. 


\subsection{The Adjustment of the Focal Spot of the L-GRIN Microlens}

The focus becomes a tunable focal spot at a relatively higher average velocity. The adjustment of the focal spot of the L-GRIN microlens can also be achieved by changing the flow rate of the liquids when the average velocity is higher. The case of no relative slipbetween the core and cladding liquids with a lower average velocity has been analyzed above. Analyzing a more sophisticated case will be helpful to better understand the influence of flow rate on the refractive index profile. For instance, the ethylene glycol solution is injected in different flow rates with DI water, andthere is a relative slip between the core and cladding liquids. For analyzing the influence of the relative slip between the core and cladding liquids on the refractive index profile, the cladding flow rate was kept constant $\left(40 \times 10^{3} \mathrm{pL} / \mathrm{s}\right)$ and the flow rate of the core inlet varied from $2 \times 10^{3} \mathrm{pL} / \mathrm{s}$ to $50 \times 10^{3} \mathrm{pL} / \mathrm{s}$ by $5 \times 10^{3} \mathrm{pL} / \mathrm{s}$. Figure 6 a shows the refractive index profile with the core inlet flow rate of $25 \times 10^{3} \mathrm{pL} / \mathrm{s}$, and the cross-sectional refractive index profiles at five different locations $(x=50,100,150,200$ and $250 \mu \mathrm{m})$ are shown in Figure 6b. It is found that there is little variation of the refractive index in central regions with different cross-sectional lengths along the $x$ axis. The width in the central region along the $y$ axis, in which the refractive index keeps almost constant, is defined as the core width. When the core inlet flow rate is $Q_{\text {core }}=25 \times 10^{3} \mathrm{pL} / \mathrm{s}$, the core width of the refractive index is $24 \mu \mathrm{m}$, as shown in Figure $6 \mathrm{~b}$. The constant refractive index in the central region hasno obvious effect on the focusing process; thus, the size of the output beam spot can be determined mainly by the core width. The curve with circle in Figure $6 \mathrm{c}$ shows the relationship between the core inlet flow rate and the core width. Additionally, the curve width dot shows the changing trend of half the width of the refrative index profile with different $Q_{\text {core }}$. When the core inlet flow rate remainsconstant, with a relatively low core inlet flow rate (lower than $10 \times 10^{3} \mathrm{pL} / \mathrm{s}$ ), the core width approachs zero. In that case, the L-GRIN microlens can theoretically focus the beam onto one point when the ratio of the core inlet flow rate to the cladding inlet flow rate is lower than 0.25 . However, the core width increases along with the increaseof the core inlet flow rate (higher than $10 \times 10^{3} \mathrm{pL} / \mathrm{s}$ ); that is, when increasing the ratio of the flow rates of the ethylene glycol solution to the DI water, the size of the output spot may continue to grow. Thus, controlling the ratio of the core inlet flow rate to the cladding inlet flow rate is a useful way to dynamically adjust the size of the light beam in microscale.
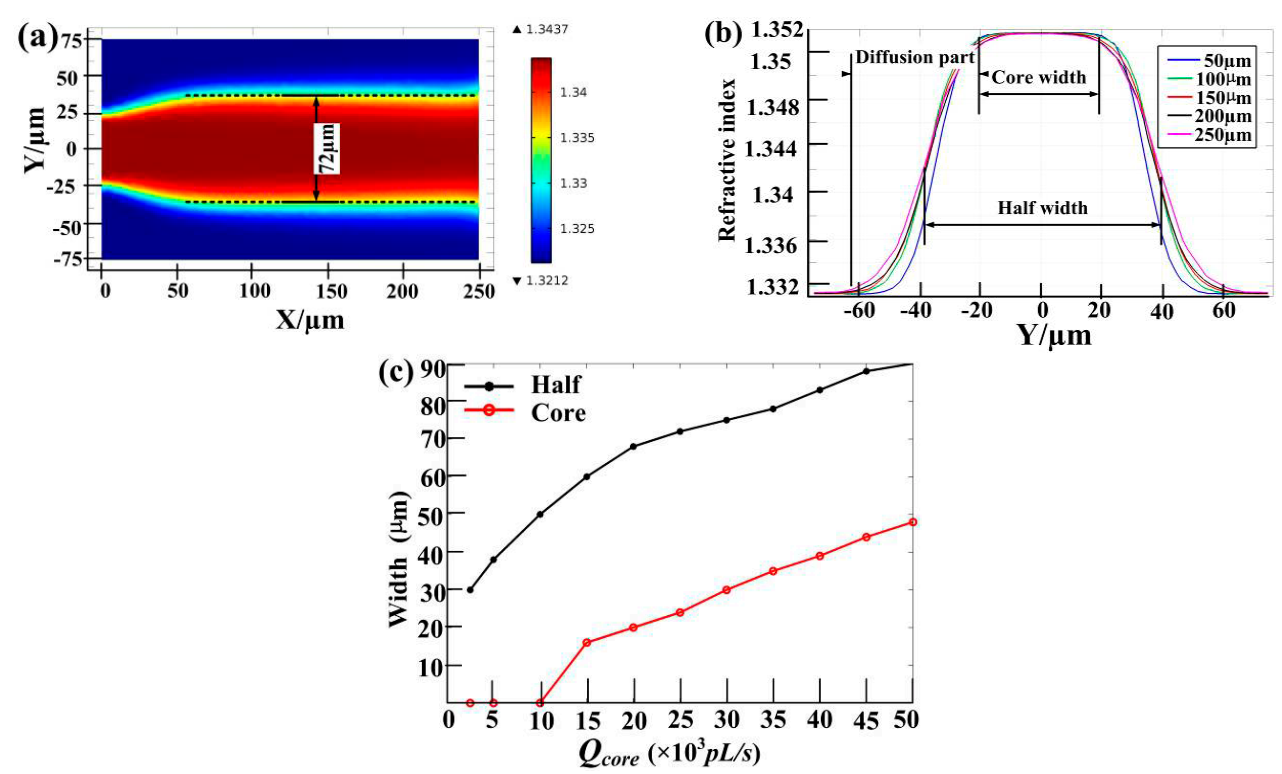

Figure 6. (a) The refractive index profile in the $x o y$ plane with $Q_{\text {core }}=25 \times 10^{3} \mathrm{pL} / \mathrm{s}$; (b) The cross-sectional refractive index profiles at five different locations $(x=50,100,150,200$ and $250 \mu \mathrm{m})$ at $Q_{\text {core }}=25 \times 10^{3} \mathrm{pL} / \mathrm{s}$; (c) The variation of core width and half width with different $Q_{\text {core }}$. 
Figure $6 \mathrm{~b}$ reveals that the cross-sectional refractive index profile in the yoz plane changes slightly even though $x$ is different, which is fitted using six-degree polynomial functions. The optimal parameters of the functions areselected through the test of the partial fitting square sum on the basis of the variance analysis of the fitting equation. For example, the fitted refractive index profile at $x=125 \mu \mathrm{m}$ with $Q_{\text {core }}=2.5 \times 10^{4} \mathrm{pL} / \mathrm{s}$ and $Q_{\text {core }}=4 \times 10^{4} \mathrm{pL} / \mathrm{s}$ can be expressed as:

$$
n=1.1 \times 10^{-12} s^{6}-2.2 \times 10^{-13} s^{5}-6.45 \times 10^{-9} s^{4}-2.73 \times 10^{-10} s^{3}+3.62 \times 10^{-6} s^{2}+1.99 \times 10^{-6} s+1.3533
$$

where $s=\sqrt{y^{2}+z^{2}}$ This is a six-order polynomial of $s$, which represents the non-linear relation between the refractive index $n$ of the liquids and the chamber position in the $x$ direction. Figure $7 \mathrm{a}$ shows that the fitting refractive index profile matches well with the simulated data. For analyzing the beam focusing effect of the L-GRIN microlens, the beam transmission and focusing process was simulated using the ray tracing method according to the refractive index profile, which is shown in Figure $7 \mathrm{~b}$. A beam spot with the diameter of $23.5 \mu \mathrm{m}$ and the focal length of $235.3 \mu \mathrm{m}$ was achieved. The ability of the tunable focusing of the beam is significant for a wide range of applications in integrated optics and lab-on-a-chip systems.
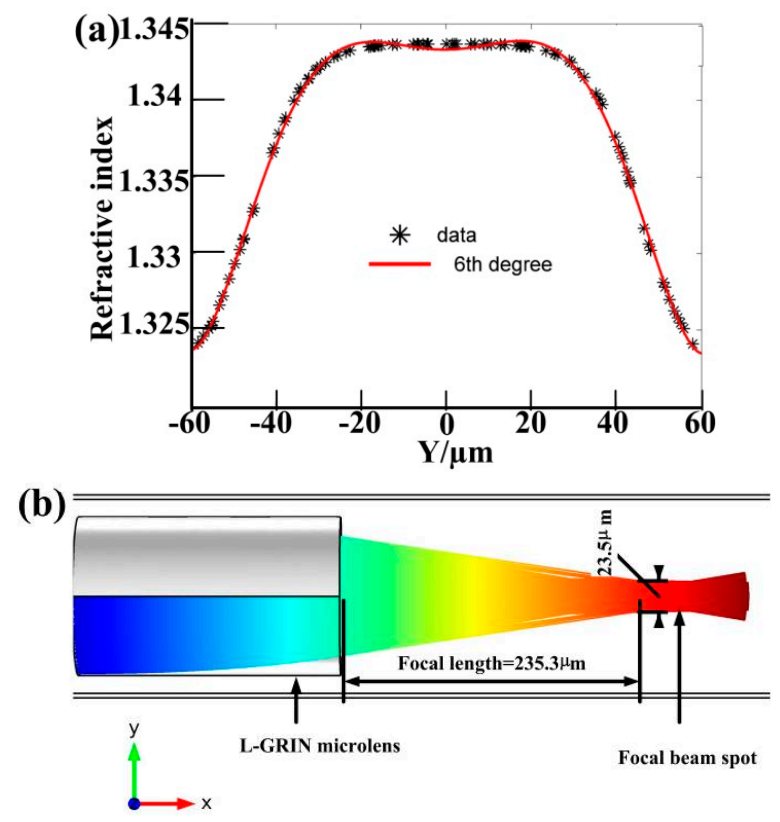

Figure 7. (a) The fitting refractive index profile of the simulated data and six-degree polynomial at $x=125 \mu \mathrm{m}$ with $Q_{\text {core }}=2.5 \times 10^{4}$ and $Q_{\text {core }}=4 \times 10^{4} \mathrm{pL} / \mathrm{s}$; $(\mathbf{b})$ Beam transmission and focusing process.

In the L-GRIN microlens, the diameter of the focal spot is affected by the focal length and the wavelength of incident light. For a given incident light, one can conclude that the focal spot is the smallest when the focal length is the shortest. As the wavelength increases, the focal length is gradually increased, and the focal spot is increased.

These discussions above are all for a steady-state flow. However, the response time of the focal length and the focal spot change are important parameters for the stability of an adaptive lens operation, which is mainly dependent on the diffusion speed of two inlet flows. According to Equation (1), the response time varies with the concentration $C$ and the diffusion coefficient $D$ of the liquids, which are also found to be affected by the temperature, the average velocity $U$ and the viscosity of the liquids, the structure and materials of the chamber, etc. For the fixed inlet liquids and the chamber, as the temperature increases, the response time decreases. However, increasing the average velocity of the liquids would lend to an unfavorable slow response time because the 
contacting time of the two liquids is shortened. Therefore, for practical applications we need to choose the suitable liquids and optimize the microlens parameters; meanwhile, the microlens material should be carefully chosen.

\section{Conclusions}

We designed an in-plane L-GRIN microlens that can realize two-dimensional light beam focusing dynamically. The diffusion coefficient, mass fraction and flow rate of the core inlet and the cladding inlet are demonstrated to be the main influencing factors for changing the refractive index profile of the L-GRIN microlens, and the influences of these factors on the focusing process of the L-GRIN microlens are studied. It is found that adjusting the mass fraction and flow rate of the ethylene glycol is an efficient way to change the focal length of the output beam. The focal length varies from 942 to $11 \mu \mathrm{m}$ at the mass fraction of ethylene glycol varying from 0.05 to 0.4 . In addition, by varying the core and cladding inlet flow rate from $0.5 \times 10^{3}$ to $5 \times 10^{3} \mathrm{pL} / \mathrm{s}$, the focal length of the microlens changes from 127.1 to $8 \mu \mathrm{m}$. In addition, the adjustable size of the output beam spot can be provided by controlling the relative slip between the core inlet and the cladding inlet flow rate with a relatively high average velocity. The flexible properties of manipulating the light beam in microscale have advantages for integrated devices in lab-on-a-chip applications.

Acknowledgments: This work is supported by the National Natural Science Foundation of China (Grant No. 61172081) and the Natural Science Foundation of Zhejiang Province, China (Grant No. LZ13F010001).

Author Contributions: Zichun Le and Yunli Sun planned and performed the reported designs and their analysis. Ying Du has written the main manuscript and prepared the figures and tables. All authors reviewed the manuscript.

Conflicts of Interest: The authors declare no conflict of interest.

\section{References}

1. Erickson, D.; Yang, C.; Psaltis, D. Optofluidics emerges from the laboratory. Photon. Spectra 2008, 42, 74-79.

2. Mao, X.L.; Lin, S.C.S.; Lapsley, M.I.; Shi, J.J.; Juluri, B.K.; Huang, T.J. A tunable optofluidic microlens based on gradient refractive index. Lab Chip 2009, 9, 2050-2058. [CrossRef] [PubMed]

3. Godin, J.; Lien, V.; Lo, Y.H. Demonstration of two-dimensional fluidic lens for integration into microfluidic flow cytometers. Appl. Phys. Lett. 2006, 89, 061106. [CrossRef]

4. Wang, Z.; el-Ali, J.; Engelund, M.; Gotsaed, T.; Perch-Nielsen, I.R.; Mogensen, K.B.; Snakenborg, D.; Kutter, J.P.; Wolff, A. Measurements of scattered light on a microchip flow cytometer with integrated polymer based optical elements. Lab Chip 2004, 4, 372-377. [CrossRef] [PubMed]

5. Martini, J.; Recht, M.I.; Huck, M.; Bern, M.W.; Johnson, N.M.; Kiesel, P. Time encoded multicolour fluorescence detection in a microfluidic flow cytometer. Lab Chip 2012, 12, 5057-5062. [CrossRef] [PubMed]

6. Yin, D.; Lunt, E.J.; Rudenko, M.I.; Deamer, D.W.; Hawkinsand, A.R.; Schmidt, H. Tailoring the transmission of liquid-core waveguides for wavelength filtering on a chip. Lab Chip 2007, 7, 1171-1175. [CrossRef] [PubMed]

7. Ozcelik, D.; Phillips, B.S.; Parks, J.W.; Measor, P.; Gulbransen, D.; Hawkins, A.R.; Schmidt, H. Dual-core optofluidic chip for independent particle detection an tunable spectral filtering. Lab Chip 2012, 12, 3728-3733. [CrossRef] [PubMed]

8. Kuiper, S.; Hendriks, B.H.W. Variable-focus liquid lens for miniature cameras. Appl. Phys. Lett. 2004, 85, 1128-1130. [CrossRef]

9. Ren, H.W.; Wu, S.T. Variable-focus liquid lens. Opt. Express 2007, 15, 5931-5936. [CrossRef] [PubMed]

10. Ren, H.W.; Wu, S.T. Tunable-focus liquid microlens array using dielectrophoretic effect. Opt. Express 2008, 16, 2646-2652. [CrossRef] [PubMed]

11. Ren, H.W.; Xianyu, H.Q.; Xu, S.; Wu, S.T. Adaptive dielectric liquid lens. Opt. Express 2008, 16, 14954-14960. [CrossRef] [PubMed]

12. Dong, L.; Agarwal, A.K.; Beebe, D.J.; Jiang, H. Adaptive liquid microlenses activated by stimuli-responsive hydrogels. Nature 2006, 442, 551-554. [CrossRef] [PubMed] 
13. Chronis, N.; Liu, G.; Jeong, K.H.; Lee, L. Tunable liquid-filled microlens array integrated with microfluidic network. Opt. Express 2003, 11, 2370-2378. [CrossRef] [PubMed]

14. Pang, L.; Levy, U.; Campbell, K.; Groisman, A.; Fainman, Y. A set of two orthogonal adaptive cylindrical lenses in a monolith elastomer device. Opt. Express 2005, 13, 9003-9013. [CrossRef] [PubMed]

15. Tang, S.K.Y.; Stan, C.A.; Whitesides, G.M. Dynamically reconfigurable liquid-core liquid-cladding lens in a microfluidic channel. Lab Chip 2008, 8, 395-401. [CrossRef] [PubMed]

16. Zickar, M.; Noell, W.; Marxer, C.; de Rooij, N. MEMS compatible micro-GRIN lenses for fiber to chip coupling of light. Opt. Express 2006, 14, 4237-4249. [CrossRef] [PubMed]

17. Huang, H.; Mao, X.L.; Lin, S.C.S.; Kiraly, B.; Huang, Y.P.; Huang, T.J. Tunable Two-Dimensional Liquid Gradient Refractive Index (L-GRIN) Lens for Variable Light Focusing. Lab Chip 2010, 10, 2387-2393. [CrossRef] [PubMed]

18. Li, Z.; Zhang, Z.; Scherer, A.; Psaltis, D. Mechanically tunable optofluidic distributed feedback dye laser. Opt. Express 2006, 14, 10494-10499. [CrossRef] [PubMed]

19. Wolfe, D.B.; Conroy, R.S.; Garstecki, P.; Mayers, B.T.; Fischbach, M.A.; Paul, K.E.; Prentiss, M.; Whitesides, G.M. Dynamic control of liquid-core/liquid-cladding optical waveguides. Proc. Natl. Acad. Sci. USA 2004, 101, 12434-12438. [CrossRef] [PubMed]

20. Perumal, M.; Raju, R.K.G. Approximate Convection-Diffusion Equations. J. Hydrol. Eng. 1999, 4, 160-164. [CrossRef]

21. Wu, Z.; Nguyen, N.T. Hydrodynamic focusing in microchannels under consideration of diffusive dispersion: Theories and experiments. Sens. Actuators B 2005, 107, 965-974. [CrossRef]

22. Kapur, J.N.; Shukla, J.B. Flow of incompressible immiscible fluids between two plates. Appl. Sci. Res. A 1964, 13, 55-60. [CrossRef]

23. Yang, Y.; Liu, A.Q.; Chin, L.K.; Zhang, X.M.; Tsai, D.P.; Lin, C.L.; Lu, C.; Wang, G.P.; Zheludev, N.I. Optofluidic waveguide as a transformation optics device for lightwave bending and manipulation. Nat. Commun. 2012, 3, 651. [CrossRef] [PubMed]

24. Ternstrom, G.; Sjostrand, A.; Aly, G.; Jernqvist, A.J. Mutual diffusion coefficients of water + ethylene glycol and water + glycerol mixtures. Chem. Eng. Data 1996, 41, 876-879. [CrossRef]

25. Wang, M.H. Measurement of Binary Mutual Diffusion Coefficient of Several Aqueous Glycol Solutions. Master's Thesis, Chung Yuan Christian University, Taiwan, July 2009. (In Chinese)

(C) 2015 by the authors; licensee MDPI, Basel, Switzerland. This article is an open access article distributed under the terms and conditions of the Creative Commons by Attribution (CC-BY) license (http://creativecommons.org/licenses/by/4.0/). 